\title{
STAR PRODUCTS AND APPLICATIONS
}

\author{
MARI IIDA AND AKIRA YOSHIOKA
}

Communicated by Ivaïlo M. Mladenov

\begin{abstract}
Star products parametrized by complex matrices are defined. Especially commutative associative star products are treated, and star exponentials with respect to these star products are considered. Jacobi's theta functions are given as infinite sums of star exponentials. As application, several concrete identities are obtained by properties of the star exponentials.
\end{abstract}

\section{Star Products}

Using an arbitrary complex symmetric matrix, we can define a star product, which gives a family of star products parameterized by complex matrices [4-6]. In particular for symmetric matrices we obtain a family of commutative associative star products $[1,2]$.

In this note, as a special case we consider a family of star product algebras of functions of one variable. Using star exponentials of these algebras we describe Jacobi's theta and its basic identities (cf. $[1,2,6]$ ).

First we consider a star product given by an arbitrary complex matrix. For simplicity, we consider star products of two variables $\left(u_{1}, u_{2}\right)$. The general case for $\left(u_{1}, u_{2}, \cdots, u_{2 m}\right)$ is similar.

For any $2 \times 2$ complex matrix $\Lambda=\left(\begin{array}{ll}\lambda_{11} & \lambda_{12} \\ \lambda_{21} & \lambda_{22}\end{array}\right) \in M_{2}(\mathbb{C})$, we have a biderivation in the space of polynomials

$$
p_{1} \overleftarrow{\partial} \Lambda \vec{\partial} p_{2}=p_{1}\left(\sum_{\alpha \beta} \lambda_{\alpha \beta} \overleftarrow{\partial_{\alpha}} \overrightarrow{\partial_{\beta}}\right) p_{2}=\sum_{\alpha \beta} \lambda_{\alpha \beta} \partial_{\alpha} p_{1} \partial_{\beta} p_{2}, \quad p_{1}, p_{2} \in \mathcal{P}\left(\mathbb{C}^{2}\right)
$$

Then we define a star product by the formula

$$
\begin{aligned}
p_{1} *_{\Lambda} p_{2} & =p_{1} \exp \left(\frac{\mathrm{i} \hbar}{2} \overleftarrow{\partial} \Lambda \vec{\partial}\right) p_{2}=p_{1}\left(\sum_{n=0}^{\infty} \frac{1}{n !}\left(\frac{\mathrm{i} \hbar}{2}\right)^{n}(\overleftarrow{\partial} \Lambda \vec{\partial})^{n}\right) p_{2} \\
& =\sum_{n=0}^{\infty} \frac{1}{n !}\left(\frac{\mathrm{i} \hbar}{2}\right)^{n} p_{1}(\overleftarrow{\partial} \wedge \vec{\partial})^{n} p_{2}
\end{aligned}
$$


where $(\overleftarrow{\partial} \Lambda \vec{\partial})^{k}$ is the $k$-th power of the derivation such that

$$
\begin{aligned}
p_{1}(\overleftarrow{\partial} \Lambda \vec{\partial})^{k} p_{2} & =p_{1} \underbrace{(\overleftarrow{\partial} \Lambda \vec{\partial}) \cdots(\overleftarrow{\partial} \Lambda \vec{\partial})}_{k} p_{2} \\
& =\sum \lambda_{\alpha_{1} \beta_{1}} \cdots \lambda_{\alpha_{k} \beta_{k}} \partial_{\alpha_{1}} \cdots \partial_{\alpha_{k}} p_{1} \partial_{\beta_{1}} \cdots \partial_{\beta_{k}} p_{2}
\end{aligned}
$$

In this setting we have

Proposition 1. For any $\Lambda \in M_{2}(\mathbb{C})$, the product $*_{\Lambda}$ is well-defined and associative on $\mathcal{P}\left(\mathbb{C}^{2}\right)$.

\section{Star Products on Functions}

The star products are well defined on the space of polynomials. In this section we look for its extension to certain class of functions on $\mathbb{C}^{2}$. We introduce a system of semi-norms and then its topology in $\mathcal{P}\left(\mathbb{C}^{2}\right)$. We take the completion to obtain a space of functions on which the star products are well defined. On this space we can consider star exponentials.

Now we define a topology. Let $\rho$ be a positive number. For every $s>0$ we define a semi-norm for polynomials by

$$
|p|_{s}=\sup _{u \in \boldsymbol{C}^{2}}\left|p\left(u_{1}, u_{2}\right)\right| \exp \left(-s|u|^{\rho}\right) .
$$

Then the system of semi-norms $\{|\cdot|\}_{s>0}$ defines a locally convex topology $\mathcal{T}_{\rho}$ on $\mathcal{P}\left(\boldsymbol{C}^{2}\right)$.

By taking the completion of $\mathcal{P}\left(C^{2}\right)$ with respect to the topology $\mathcal{T}_{\rho}$ we obtain the Fréchet space $\mathcal{E}_{\rho}\left(\boldsymbol{C}^{2}\right)$.

Proposition 2. For a positive number $\rho$, the Fréchet space $\mathcal{E}_{\rho}$ consists of entire functions on the complex plane $C^{2}$ with finite semi-norm for every $s>0$, namely,

$$
\mathcal{E}_{\rho}\left(\boldsymbol{C}^{2}\right)=\left\{f \in \mathcal{H}\left(\boldsymbol{C}^{2}\right) ;|f|_{s}<\infty, \text { for all } s>0\right\} .
$$

As to the continuity of star products, the space $\mathcal{E}_{\rho}\left(\boldsymbol{C}^{2}\right), 0<\rho \leq 2$ is very suitable, namely, we have the following

Theorem 3. On the space $\mathcal{E}_{\rho}\left(\boldsymbol{C}^{2}\right)$ for $0<\rho \leq 2$, every product $*_{\Lambda}$ is continuous. 
For the spaces $\mathcal{E}_{\rho}\left(\boldsymbol{C}^{2}\right)$ where $\rho>2$, the situation is not so good, but we still can rely on the following result.

Theorem 4. For $\rho>2$, take $\rho^{\prime}>0$ such that $\frac{1}{\rho^{\prime}}+\frac{1}{\rho}=1$. Then every star product $*_{\Lambda}$ defines a continuous bilinear product

$$
*_{\Lambda}: \mathcal{E}_{\rho}\left(\boldsymbol{C}^{2}\right) \times \mathcal{E}_{\rho^{\prime}}\left(\boldsymbol{C}^{2}\right) \rightarrow \mathcal{E}_{\rho}\left(\boldsymbol{C}^{2}\right), \quad \mathcal{E}_{\rho^{\prime}}\left(\boldsymbol{C}^{2}\right) \times \mathcal{E}_{\rho}\left(\boldsymbol{C}^{2}\right) \rightarrow \mathcal{E}_{\rho}\left(\boldsymbol{C}^{2}\right) .
$$

This means that $\left(\mathcal{E}_{\rho}\left(\boldsymbol{C}^{2}\right), *_{\Lambda}\right)$ is a continuous $\mathcal{E}_{\rho^{\prime}}\left(\boldsymbol{C}^{2}\right)$-bimodule.

Let us introduce the Fréchet space

$$
\mathcal{E}_{\rho+}\left(\boldsymbol{C}^{2}\right)=\cap_{\lambda>\rho} \mathcal{E}_{\lambda}\left(\boldsymbol{C}^{2}\right)
$$

and consider the exponential element

$$
\exp _{*_{\Lambda}} t\left(\frac{H}{\mathrm{i} \hbar}\right)=\sum_{n=0}^{\infty} \frac{t^{n}}{n !} \underbrace{\frac{H}{\mathrm{i} \hbar} *_{\Lambda} \cdots *_{\Lambda} \frac{H}{\mathrm{i} \hbar}}_{n}
$$

in $\mathcal{E}_{\rho}\left(C^{2}\right)$. The right hand side is not convergent in general. Hence for a polynomial $H \in \mathcal{P}\left(\boldsymbol{C}^{2}\right)$, we define the star exponential $\exp _{*_{\Lambda}} t(H / \mathrm{i} \hbar)$ by the differential equation

$$
\frac{\mathrm{d}}{\mathrm{d} t} \exp _{*_{\Lambda}} t\left(\frac{H}{\mathrm{i} \hbar}\right)=\frac{H}{\mathrm{i} \hbar} *_{\Lambda} \exp _{*} t\left(\frac{H}{\mathrm{i} \hbar}\right),\left.\quad \exp _{*_{\Lambda}} t\left(\frac{H}{\mathrm{i} \hbar}\right)\right|_{t=0}=1 .
$$

When $H \in \mathcal{P}\left(\boldsymbol{C}^{2}\right)$ is a linear element, then $\exp _{*} t\left(\frac{H}{\mathrm{i} \hbar}\right)$ belongs to the good space $\mathcal{E}_{1+}\left(\subset \mathcal{E}_{2}\right)$. In this case, the star exponentials are obtained directly by the formula $\sum_{n=0}^{\infty} \frac{t^{n}}{n !} \underbrace{\frac{H}{\mathrm{i} \hbar} *_{\Lambda} \cdots *_{\Lambda} \frac{H}{\mathrm{i} \hbar}}_{n}$.

On the other hand, we remark here that the most interesting case is given by quadratic form $H \in \mathcal{P}\left(\boldsymbol{C}^{2}\right)$, which case $\sum_{n=0}^{\infty} \frac{t^{n}}{n !} \frac{H}{\mathrm{i} \hbar} *_{\Lambda} \cdots *_{\Lambda} \frac{H}{\mathrm{i} \hbar}$ is not convergent and we need the differential equation to define the star exponentials. The star exponential belongs to the space $\mathcal{E}_{2+}\left(C^{2}\right)$, which is difficult to treat at present.

\section{Theta Functions}

In this section, we consider the star product for the simple case where

$$
\Lambda=\left(\begin{array}{ll}
\rho & 0 \\
0 & 0
\end{array}\right) \text {. }
$$


Then we can see easily that the star product is commutative and explicitly given by $p_{1} *_{\Lambda} p_{2}=p_{1} \exp \left(\frac{\mathrm{i} \hbar \rho}{2} \overleftarrow{\partial_{u_{1}}} \overrightarrow{\partial_{u_{1}}}\right) p_{2}$. This means that the algebra is essentially reduced to the space of functions of one variable $u_{1}$. Thus, we consider functions $f(w), g(w)$ of one variable $w \in \mathbb{C}$ for which we define a commutative star product $*_{\tau}$ with complex parameter $\tau$ such that

$$
f(w) *_{\tau} g(w)=f(w) \mathrm{e}^{\frac{\tau}{2} \overleftarrow{\partial} w \vec{\partial}_{w}} g(w)
$$

\subsection{Star Theta Functions}

In this section we consider the Jacobi's theta functions as an example of star exponentials.

A direct calculation gives

$$
\exp _{*_{\tau}} \mathrm{i} t w=\exp \left(\mathrm{i} t w-(\tau / 4) t^{2}\right) .
$$

Hence for $\operatorname{Re} \tau>0$, the star exponential $\exp _{*_{\tau}} n \mathrm{i} w=\exp \left(n \mathrm{i} w-(\tau / 4) n^{2}\right)$ is rapidly decreasing with respect to integer $n$ and then we can consider summations for $\tau$ satisfying $\operatorname{Re} \tau>0$

$$
\sum_{n=-\infty}^{\infty} \exp _{*_{\tau}} 2 n \mathrm{i} w=\sum_{n=-\infty}^{\infty} \exp \left(2 n \mathrm{i} w-\tau n^{2}\right)=\sum_{n=-\infty}^{\infty} q^{n^{2}} \mathrm{e}^{2 n \mathrm{i} w}, \quad q=\mathrm{e}^{-\tau} .
$$

This is Jacobi's theta function $\theta_{3}(w, \tau)$ (cf. [1]). Then we have expression of the theta functions as

$$
\begin{array}{ll}
\theta_{1 *_{\tau}}(w)=\frac{1}{\mathrm{i}} \sum_{n=-\infty}^{\infty}(-1)^{n} \exp _{*_{\tau}}(2 n+1) \mathrm{i} w, & \theta_{2 *_{\tau}}(w)=\sum_{n=-\infty}^{\infty} \exp _{*_{\tau}}(2 n+1) \mathrm{i} w \\
\theta_{3 *_{\tau}}(w)=\sum_{n=-\infty}^{\infty} \exp _{*_{\tau}} 2 n \mathrm{i} w, & \theta_{4 *_{\tau}}(w)=\sum_{n=-\infty}^{\infty}(-1)^{n} \exp _{*_{\tau}} 2 n \mathrm{i} w .
\end{array}
$$

Remark that $\theta_{k *_{\tau}}(w)$ are the Jacobi's theta functions $\theta_{k}(w, \tau), k=1,2,3,4$ respectively. This is obvious by the exponential law

$$
\begin{aligned}
2 \exp _{*_{\tau}} 2 \mathrm{i} w *_{\tau} \theta_{k *_{\tau}}(w) & =\theta_{k *_{\tau}}(w), & & k=2,3 \\
\exp _{*_{\tau}} 2 \mathrm{i} w *_{\tau} \theta_{k *_{\tau}}(w) & =-\theta_{k *_{\tau}}(w), & & k=1,4 .
\end{aligned}
$$

Then using $\exp _{*_{\tau}} 2 \mathrm{i} w=\mathrm{e}^{-\tau} \mathrm{e}^{2 \mathrm{i} w}$ and the product formula directly we have

$$
\begin{aligned}
2 \mathrm{e}^{2 \mathrm{i} w-\tau} \theta_{k *_{\tau}}(w+\mathrm{i} \tau) & =\theta_{k *_{\tau}}(w), & & k=2,3 \\
\mathrm{e}^{2 \mathrm{i} w-\tau} \theta_{k *_{\tau}}(w+\mathrm{i} \tau) & =-\theta_{k *_{\tau}}(w), & & k=1,4 .
\end{aligned}
$$


Following Toda's idea [3] we obtain the following formulas with the help of the above expressions. In what follows we use as a variable $v$ instead of $w$ given by the relation $\pi v=w$.

\section{Lemma 5.}

$$
\begin{aligned}
& \theta_{1_{* \tau}}^{2}(v)=\left(\sum_{\lambda}^{e} \sum_{\mu}^{o}-\sum_{\lambda}^{o} \sum_{\mu}^{e}\right) M(\lambda, \mu) \\
& \theta_{2_{* \tau}}^{2}(v)=\left(\sum_{\lambda}^{e} \sum_{\mu}^{o}+\sum_{\lambda}^{o} \sum_{\mu}^{e}\right) M(\lambda, \mu) \\
& \theta_{3_{*_{\tau}}}^{2}(v)=\left(\sum_{\lambda}^{e} \sum_{\mu}^{e}+\sum_{\lambda}^{o} \sum_{\mu}^{o}\right) M(\lambda, \mu) \\
& \theta_{4_{*_{\tau}}}^{2}(v)=\left(\sum_{\lambda}^{e} \sum_{\mu}^{e}-\sum_{\lambda}^{o} \sum_{\mu}^{o}\right) M(\lambda, \mu)
\end{aligned}
$$

where $M(\lambda, \mu)=\mathrm{e}^{\frac{\tau}{2}\left(\lambda^{2}-\mu^{2}\right) \pi^{2}} \mathrm{e}_{*_{\tau}}^{2 \lambda \pi \mathrm{i} v}$ and $\sum_{\lambda}^{e}$ means that $\lambda$ runs through all even integers etc.

Proof: By a direct calculation we have

$$
\theta_{1_{*}}^{2}(v)=-\sum_{n, m}(-1)^{n+m} \mathrm{e}_{*_{\tau}}^{(2 n+1) \pi \mathrm{i} v} \mathrm{e}_{*_{\tau}}^{(2 m+1) \pi \mathrm{i} v} .
$$

We notice that

$$
\begin{aligned}
\mathrm{e}_{*_{\tau}}^{(2 n+1) \pi \mathrm{i} v} \mathrm{e}_{*_{\tau}}^{(2 m+1) \pi \mathrm{i} v} & =-\mathrm{e}^{-\frac{\tau}{2}(2 n+1) \pi \mathrm{i}(2 m+1) \pi \mathrm{i}} \mathrm{e}_{*_{\tau}}^{(2 n+1) \pi \mathrm{i} v} *_{\tau} \mathrm{e}_{*_{\tau}}^{(2 m+1) \pi \mathrm{i} v} \\
& =-\mathrm{e}^{\frac{\tau}{2}(2 n+1)(2 m+1) \pi^{2}} \mathrm{e}_{*_{\tau}}^{2(n+m+1) \pi \mathrm{i} v}
\end{aligned}
$$

The introduction of $\lambda=n+m+1$ and $\mu=n-m$ gives after some work the following formula

$$
\theta_{1_{* \tau}}^{2}(v)=\sum_{\lambda, \mu}(-1)^{\lambda} \mathrm{e}^{\frac{\tau}{2}\left(\lambda^{2}-\mu^{2}\right) \pi^{2}} \mathrm{e}_{*_{\tau}}^{2 \lambda \pi \mathrm{i} v}
$$

Cancellation in the summation yields

$$
\theta_{1_{* \tau}}^{2}(v)=\left(\sum_{\lambda}^{e} \sum_{\mu}^{o}-\sum_{\lambda}^{o} \sum_{\mu}^{e}\right) \mathrm{e}^{\frac{\tau}{2}\left(\lambda^{2}-\mu^{2}\right) \pi^{2}} \mathrm{e}_{*_{\tau}}^{2 \lambda \pi \mathrm{i} v}
$$


which produces the desired result. Other identities are obtained in a similar manner.

Using Lemma 5 we easily obtain

\section{Proposition 6.}

$$
\theta_{1_{* \tau}}^{4}(v)+\theta_{3_{* \tau}}^{4}(v)=\theta_{2_{* \tau}}^{4}(v)+\theta_{4_{* \tau}}^{4}(v)
$$

Proof: We have

$$
\begin{aligned}
\theta_{1_{*_{\tau}}}^{4}(v)= & \left(\sum_{l}^{e} \sum_{k}^{o}-\sum_{l}^{o} \sum_{k}^{e}\right) M(l, k)\left(\sum_{\lambda}^{e} \sum_{\mu}^{o}-\sum_{\lambda}^{o} \sum_{\mu}^{e}\right) M(\lambda, \mu) \\
= & \left(\sum_{l}^{e} \sum_{k}^{o} M(l, k)-\sum_{l}^{o} \sum_{k}^{e} M(l, k)\right)\left(\sum_{\lambda}^{e} \sum_{\mu}^{o} M(\lambda, \mu)\right. \\
& \left.-\sum_{\lambda}^{o} \sum_{\mu}^{e} M(\lambda, \mu)\right)=(A-B)(C-D) .
\end{aligned}
$$

Similarly we have $\theta_{2_{* \tau}}^{4}(v)=(A+B)(C+D)$. By the same manner we see

$$
\begin{aligned}
\theta_{3_{* \tau}}^{4}(v) & =\left(\sum_{l}^{e} \sum_{k}^{e}+\sum_{l}^{o} \sum_{k}^{o}\right) M(l, k)\left(\sum_{\lambda}^{e} \sum_{\mu}^{e}+\sum_{\lambda}^{o} \sum_{\mu}^{o}\right) M(\lambda, \mu) \\
& =(E+F)(G+H)
\end{aligned}
$$

and $\theta_{4_{* \tau}}^{4}(v)=(E-F)(G-H)$. Therefore

$$
\begin{aligned}
\theta_{1_{* \tau}}^{4}(v) & +\theta_{3_{* \tau}}^{4}(v)-\left(\theta_{2_{* \tau}}^{4}(v)+\theta_{0_{* \tau}}^{4}(v)\right)=(A-B)(C-D) \\
& +(E+F)(G+H)-\{(A+B)(C+D)+(E-F)(G-H)\} \\
= & 2(-A D-B C+E H+F G) .
\end{aligned}
$$

Arranging the summation we have

$$
\begin{aligned}
2 & \left(-\sum_{l}^{e} \sum_{k}^{o} \sum_{\lambda}^{o} \sum_{\mu}^{e}-\sum_{l}^{o} \sum_{k}^{e} \sum_{\lambda}^{e} \sum_{\mu}^{o}+\sum_{l}^{e} \sum_{k}^{e} \sum_{\lambda}^{o} \sum_{\mu}^{o}+\sum_{l}^{o} \sum_{k}^{o} \sum_{\lambda}^{e} \sum_{\mu}^{e}\right) \\
& \times \mathrm{e}^{\frac{\tau}{2}\left(l^{2}-k^{2}+\lambda^{2}-\mu^{2}\right) \pi^{2}} \mathrm{e}_{*_{\tau}}^{2 \pi \mathrm{i} v} \mathrm{e}_{*_{\tau}}^{2 \lambda \pi \mathrm{i} v} \\
= & 2\left(\sum_{l}^{o} \sum_{\lambda}^{e}\left(\sum_{k}^{o} \sum_{\mu}^{e}-\sum_{k}^{e} \sum_{\mu}^{o}\right)-\sum_{l}^{e} \sum_{\lambda}^{o}\left(\sum_{k}^{o} \sum_{\mu}^{e}-\sum_{k}^{e} \sum_{\mu}^{o}\right)\right) \\
& \times \mathrm{e}^{\frac{\tau}{2}\left(l^{2}-k^{2}+\lambda^{2}-\mu^{2}\right) \pi^{2}} \mathrm{e}_{*_{\tau}}^{2 \pi \mathrm{i} v} \mathrm{e}_{*_{\tau}}^{2 \lambda \pi \mathrm{i} v}=0
\end{aligned}
$$


since $\sum_{k}^{o} \sum_{\mu}^{e} \mathrm{e}^{-\frac{\tau}{2}\left(k^{2}+\mu^{2}\right)}=\sum_{k}^{e} \sum_{\mu}^{o} \mathrm{e}^{-\frac{\tau}{2}\left(k^{2}+\mu^{2}\right)}$.

Proposition 7. For a complex parameter $a \in \mathbb{C}$ we have the identity

$$
\theta_{3_{*} \tau}^{2}(v) \theta_{3_{*_{\tau}}}^{2}(a)+\theta_{1_{* \tau}}^{2}(v) \theta_{1_{* \tau}}^{2}(a)=\theta_{3_{* \tau}}^{2}(0) \theta_{3_{* \tau}}(v+a) \theta_{3_{* \tau}}(v-a) .
$$

Proof: By a similar manner as in Lemma 5 we have

$$
\theta_{3_{* \tau}}(v+a) \theta_{3_{* \tau}}(v-a)=\left(\sum_{l}^{e} \sum_{\lambda}^{e}+\sum_{l}^{o} \sum_{\lambda}^{o}\right) \mathrm{e}^{\frac{\tau}{2}\left(l^{2}-\lambda^{2}\right) \pi^{2}} \mathrm{e}^{2 \lambda \pi \mathrm{i} a} \mathrm{e}_{*_{\tau}}^{2 l \pi \mathrm{i} v} .
$$

Since $\left.\mathrm{e}_{*_{\tau}}^{2 \lambda \pi \mathrm{i} v}\right|_{v=0}=\left.\mathrm{e}^{-\tau \lambda^{2} \pi^{2}} \mathrm{e}^{2 \lambda \pi \mathrm{i} v}\right|_{v=0}=\mathrm{e}^{-\tau \lambda^{2} \pi^{2}}$ we have also

$$
\begin{aligned}
& \theta_{3_{* \tau}}^{2}(0)=\left(\sum_{k}^{e} \sum_{\mu}^{e}+\sum_{k}^{o} \sum_{\mu}^{o}\right) \mathrm{e}^{-\frac{\tau}{2}\left(k^{2}+\mu^{2}\right) \pi^{2}} \\
& \theta_{3_{* \tau}}^{2}(a)=\left(\sum_{k}^{e} \sum_{\mu}^{e}+\sum_{k}^{o} \sum_{\mu}^{o}\right) \mathrm{e}^{-\frac{\rho}{2}\left(k^{2}+\mu^{2}\right) \pi^{2}} \mathrm{e}^{2 \lambda \pi \mathrm{i} a} \\
& \theta_{1_{* \tau}}^{2}(a)=\left(\sum_{\lambda}^{e} \sum_{\mu}^{o}-\sum_{\lambda}^{o} \sum_{\mu}^{e}\right) \mathrm{e}^{-\frac{\tau}{2}\left(\lambda^{2}+\mu^{2}\right) \pi^{2}} \mathrm{e}^{2 \lambda \pi \mathrm{i} a} .
\end{aligned}
$$

Then we obtain

$$
\begin{aligned}
\theta_{3_{*_{\tau}}}^{2} & (v) \theta_{3_{* \tau}}^{2}(a)+\theta_{1_{*_{\tau}}}^{2}(v) \theta_{1_{*_{\tau}}}^{2}(a) \\
= & \left(\sum_{l}^{e} \sum_{k}^{e} \sum_{\lambda}^{e} \sum_{\mu}^{e}+\sum_{l}^{e} \sum_{k}^{e} \sum_{\lambda}^{o} \sum_{\mu}^{o}+\sum_{l}^{o} \sum_{k}^{o} \sum_{\lambda}^{e} \sum_{\mu}^{e}+\sum_{l}^{o} \sum_{k}^{o} \sum_{\lambda}^{o} \sum_{\mu}^{o}\right. \\
& \left.+\sum_{l}^{e} \sum_{k}^{o} \sum_{\lambda}^{e} \sum_{\mu}^{o}-\sum_{l}^{e} \sum_{k}^{o} \sum_{\lambda}^{o} \sum_{\mu}^{e}-\sum_{l}^{o} \sum_{k}^{e} \sum_{\lambda}^{e} \sum_{\mu}^{o}+\sum_{l}^{o} \sum_{k}^{e} \sum_{\lambda}^{o} \sum_{\mu}^{e}\right) \\
& \left.\times \mathrm{e}^{\frac{\tau}{2}\left(l^{2}-\lambda^{2}-k^{2}-\mu^{2}\right) \pi^{2}} \mathrm{e}^{2 \lambda \pi \mathrm{i} a} \mathrm{e}_{*_{\tau}}^{2 l \pi \mathrm{i} v}\right) \\
= & \left(\sum_{k}^{e} \sum_{\mu}^{e}+\sum_{k}^{o} \sum_{\mu}^{o}\right) \mathrm{e}^{-\frac{\tau}{2}\left(k^{2}+\mu^{2}\right) \pi^{2}}\left(\sum_{l}^{e} \sum_{\lambda}^{e}+\sum_{l}^{o} \sum_{\lambda}^{o}\right) \\
& \times \mathrm{e}^{\frac{\tau}{2}\left(l^{2}-\lambda^{2}\right) \pi^{2}} \mathrm{e}^{2 \lambda \pi \mathrm{i} a} \mathrm{e}_{*_{\rho}}^{2 l \pi \mathrm{i} v}=\theta_{3_{* \tau}}^{2}(0) \theta_{3_{* \tau}}(v+a) \theta_{3_{* \tau}}(v-a) .
\end{aligned}
$$




\section{Acknowledgements}

The second author is partially supported by the Grant-in-Aid for Scientific Research (\# 21540096). The authors are thankful to Professors Ivaïlo Mladenov and Gaetano Vilasi for discussions and encouragement in Tokyo. The second author is grateful for the hospitality of the Institute of Biophysics of the Bulgarian Academy of Science during his stay there.

\section{References}

[1] Omori H, Maeda Y., Miyazaki N. and Yoshioka A., Orderings and Nonformal Deformation Quantization, Lett. Math. Phys. 82 (2007) 153-175.

[2] Omori H, Maeda Y., Miyazaki N. and Yoshioka A., Deformation of Expression of Algebras, (preprint)

[3] Toda M., Introduction to Elliptic Functions (in Japanese), Nihon Hyoronshya, Tokyo, 2001.

[4] Tomihisa T. and Yoshioka A., Star Products and Star Exponentials, J. Geom. Symm. Phys. 19 (2010) 99-111.

[5] Yoshioka A., A Family of Star Products and its Application, In: Proceedings XXVI Workshop on Geometrical Methods in Physics, P. Kielanowski, A. Odzijewicz, M. Schlichenmaier and T. Voronov (Eds), AIP Conference Proceedings vol. 956, Melville, New York 2007, pp 37-42.

[6] Yoshioka A., Examples of Star Exponentials, In: Proceedings XXVIII Workshop on Geometrical Methods in Physics, P. Kielanowski, S. T. Ali, A. Odzijewicz, M. Schlichenmaier and T. Voronov (Eds), AIP Conference Proceedings vol. 1191, Melville, New York 2009, pp 188-193.

Mari Iida

Department of Mathematics

Tokyo University of Science

162-8601 Tokyo

JAPAN

E-mail address: pochi_mary@hotmail.com

Akira Yoshioka

Department of Mathematics

Tokyo University of Science

162-8601 Tokyo

JAPAN

E-mail address: yoshioka@rs.kagu.tus.ac.jp 